\title{
HEXAGONAL PATTERNS IN A MODEL FOR ROTATING CONVECTION
}

\author{
S. MADRUGA* and C. PÉREZ-GARCÍA ${ }^{\dagger}$ \\ Instituto de Física, Universidad de Navarra, \\ E-31080 Pamplona, Navarra, Spain \\ * Institut de Physique, Université de Liège, \\ B5, Sart-Tilman, B-4000 Liège, Belgium \\ ${ }^{\dagger}$ Depto. Ingeniería Mecánica, E. T. S. Ingenieros (TECNUN), \\ Universidad de Navarra, E-20018 San Sebastián, Spain
}

\begin{abstract}
We study a model equation that mimics convection under rotation in a fluid with temperaturedependent properties (non-Boussinesq (NB)), high Prandtl number and idealized boundary conditions. It is based on a model equation proposed by Segel [1965] by adding rotation terms that lead to a Küppers-Lortz instability [Küppers \& Lortz, 1969] and can develop into oscillating hexagons. We perform a weakly nonlinear analysis to find out explicitly the coefficients in the amplitude equation as functions of the rotation rate. These equations describe hexagons and oscillating hexagons quite well, and include the Busse-Heikes (BH) model [Busse \& Heikes, 1980] as a particular case. The sideband instabilities as well as short wavelength instabilities of such hexagonal patterns are discussed and the threshold for oscillating hexagons is determined.
\end{abstract}

Keywords: Rotating convection; pattern formation; hydrodynamic instability.

\section{Introduction}

In the last decades Rayleigh-Bénard convection has been the canonical system in studying spatiotemporal patterns. More recently the interest in rotating convection was motivated by the dynamics of planetary and stellar atmospheres and the circulation of ocean currents. In addition, Coriolis forces perturb the fluid velocity and new features, absent in the nonrotating case, appear in the system. For example, spatiotemporal chaos can be obtained immediately above threshold where the small amplitude of the pattern allows a perturbative analysis [Bodenschatz et al., 2000]. The main control parameter then is the rotation rate $\Omega$. When $\Omega$ is greater than a critical value $\Omega_{c}$ an ideal pattern of straight convection rolls loses its stability through the socalled Küppers-Lortz (KL) instability [Küppers \& Lortz, 1969]. The mechanism of this instability is that rolls become unstable with respect to another set of rolls with their axis rotated by an angle $\theta_{\mathrm{KL}}$ from the original set. The new set will in turn become unstable towards rolls rotated at a further $\theta_{\mathrm{KL}}$, and so on, hence there is no saturated steadystate pattern.

As the local orientation of rolls is switched by $\theta_{\mathrm{KL}} \approx 2 \pi / 3$, Busse and Heikes (BH) [Busse \& Heikes, 1980] proposed a reduced model consisting of three coupled equations in the rotating frame, that captures some important features of the KL instability. However, experiments showed chaotically time-dependent coexistence of domains with different orientations [Bodenschatz et al., 2000]. Indeed, Tu and Cross [Tu \& Cross, 1992; Cross et al., 1994] extended the BH model by adding spatial gradient terms and thus proposed three coupled amplitude equations as a theoretical model. Numerical computations of the model show spatial patterns that 
seem dominated by domain wall motions, in agreement with experiments.

Unfortunately, the model in reference $[\mathrm{Tu} \&$ Cross, 1992] settles on some preferred directions, so breaking the isotropy of the system. To avoid this limitation the Swift-Hohenberg model has been generalized by adding suitable nonlinear terms that break the chiral symmetry. The advantages of the Swift-Hohenberg (SH) equation are: (1) it is a scalar equation and (b) it is rotationally invariant (no prefixed orientation is required). Its main shortcoming is the fact that its coefficients are obtained from approximate expansions of coupling nonlinear terms which reproduce quite well rotating patterns of rolls [Fantz et al., 1992; Ponty et al., 1997]. Though some authors considered extensions of the SH equation in which a small quadratic term [Millán-Rodríguez et al., 1992; Sain \& Riecke, 2000] which stabilizes hexagons is included, to our knowledge this has not yet been derived from the basic hydrodynamics equations.

Physically, hexagons are obtained in convection when the up-down symmetry is broken, as it occurs, for example in Bénard and non-Boussinesq convection [Busse, 1967]. Instead of the KL instability, rotation under these circumstances will induce natural oscillations on the amplitudes of three modes at $2 \pi / 3$ that make up an hexagon [Swift, 1984; Soward, 1985]. In the present paper, we propose a model that retains the main physical contributions from rotating convection, but is much more simple to solve than the hydrodynamic equations. The model is grounded on an equation proposed by Segel [1965] to deal with NB convection. Our main aim is to derive the coefficients of the amplitude equations for rotating convection within this model, their dependence on rotation and the ensuing stability diagrams near threshold. These show that convection in a horizontal NB fluid layer rotated about the vertical axis is a good candidate for studying interesting spatiotemporal phenomena even close to threshold so that a Ginzburg-Landau description should still be valid.

The paper is organized as follows. In Sec. 2 we introduce the scalar model and set up the linear stability analysis. In Sec. 3 a weakly nonlinear analysis is completed. The resulting amplitude equations include quadratic spatial terms besides the usual diffusive one. The stability of hexagons against amplitude and phase perturbations are studied in Sec. 4 . The main conclusions and a discussion are included in the last section.

\section{Model Equation}

Some years ago Segel [1965] proposed a simplified model to illustrate nonlinear techniques without the computational drawbacks in dealing with full hydrodynamic equations. This model consists of one equation for a scalar field $W$, usually associated with the vertical velocity. It is built with the following ingredients: (1) the kernel of the hydrodynamical linear problem, (2) a minimal (quadratic) nonlinear term which represents the advective term, and (3) a term that breaks the midplane symmetry and accounts for NB convection that can give rise to hexagonal cells [Busse, 1967]. The model equation is

$\partial_{t} W-\nabla^{6} W+R \nabla^{2} W-2 \gamma(\cos \pi z) W=-W \partial_{z} W$

with the boundary conditions $W=\partial_{z}^{2} W=\partial_{z}^{4} W=$ 0 at $z=0,1$. Here $\nabla$ stands for the horizontal gradient, $R$ for the control parameter (similar to the Rayleigh number in Rayleigh-Bénard convection) and $\gamma$ for NB contributions. It is worth noticing that NB effects have been studied experimentally in different fluids [Ciliberto et al., 1988; Morris et al., 1993; Assenheimer \& Steinberg, 1996; Bajaj et al., 1997].

Following this line of thought we extend this model by including rotation. Rotation contributes to the linear kernel through a term $T^{2} \partial_{z}^{2} W$ [Chandrasekhar, 1961] which must not depend on the rotation sign. (Here $T$ represents the rotation rate, equivalent to the Taylor number in rotating convection.) We assume that rotation enters also through a quadratic term which contains spatial derivatives that break the rotation symmetry $T \mathbf{e}_{z} \cdot[\nabla W \times$ $\left.\nabla \partial_{z} W\right]$. Hence the model results in the equation

$$
\begin{aligned}
\partial_{t} W & -\nabla^{6} W+R \nabla^{2} W-T^{2} \partial_{z}^{2} W-2 \gamma(\cos \pi z) W \\
& =-W \partial_{z} W+T \mathbf{e}_{z} \cdot\left[\nabla W \times \nabla \partial_{z} W\right]
\end{aligned}
$$

The linear stability of this model is easily solved after expanding $W$ in terms of normal modes $W(x, y, z, t)=e^{\sigma t} e^{i \mathbf{k} \cdot \mathbf{x}} g(z)$ where $\sigma$ is the growth rate and $g(z)$ stands for the vertical eigenfunction. For $\gamma=0$ (Boussinesq case) $g(z)=\sin (\pi z)$, as in Rayleigh-Bénard convection under free-free boundary conditions. For the general case $\gamma \neq 0$ we take a Galerkin expansion $g(z)=A_{1} \sin (\pi z)+$ $\gamma A_{2} \sin (2 \pi z)+\cdots$ that gives the characteristic equation

$$
\begin{aligned}
\sigma= & R k^{2}-\left(\pi^{2}+k^{2}\right)^{3}-\pi^{2} T^{2} \\
& +\frac{\gamma^{2}}{\left(4 \pi^{2}+k^{2}\right)^{3}-\left(\pi^{2}+k^{2}\right)^{3}+3 \pi^{2} T^{2}}
\end{aligned}
$$


This gives a marginal stability curve $R(k, T)(\sigma=$ $0)$ that changes with rotation: the critical values $R_{c}$ and $k_{c}$ increase when $T$ increases [Chandrasekhar, 1961]. Besides the usual rotating convection part, the last expression contains small NB corrections of $O\left(\gamma^{2}\right)$, negligible in comparison with the rest of the terms and therefore not considered in the following. Consequently the Galerkin expansion of the eigenfunctions can be truncated at order two. In so doing we arrive at

$$
A_{2}=\frac{A_{1}}{\left(k^{2}+4 \pi^{2}\right)^{3}-k^{2} R_{c}+4 \pi^{2} T^{2}}
$$

Because $k_{c}(T=0)=\pi / \sqrt{2}=2.22$ and $R_{c}(T=$ $0)=1315$, the last relationship gives at most $A_{2} \approx 10^{-5} A_{1}$. Although small, the contribution of $A_{2}$ must be retained to get a hexagonal pattern.

\section{Weakly Nonlinear Analysis}

We checked the validity of the rotation terms in Eq. (2) by a stability analysis of rolls in the case of a Boussinesq fluid $(\gamma=0)$. The rolls become unstable to KL instability, i.e. rolls with a given orientation are replaced by others rotated at a certain angle at the values (see Fig. 1)

$$
\Theta_{\mathrm{KL}}=47^{\circ} \quad T_{\mathrm{KL}}=2.05
$$

in qualitative agreement with the results in [Küppers \& Lortz, 1969] and similar to that found through a SH model with a cubic rotation term
[Roxin \& Riecke, 2002]. (The discrepancy between this value and the true $\mathrm{KL}$ angle $\theta_{\mathrm{KL}}=58^{\circ}$ is not important for the ensuing study of hexagons.)

In the general case $(\gamma \neq 0)$ a multiple scale analysis of Eq. (2) around a perfect hexagon solution $W=\sum_{i=1}^{3}\left(A_{i} \exp i \mathbf{k}_{i} \cdot \mathbf{x}+\right.$ c.c. $)$ leads to

$$
\begin{aligned}
\partial_{t} A_{1}= & \mu A_{1}+v \bar{A}_{2} \bar{A}_{3}-g\left|A_{1}\right|^{2} A_{1} \\
& -h_{2}\left|A_{2}\right|^{2} A_{1}-h_{3}\left|A_{3}\right|^{2} A_{1}
\end{aligned}
$$

where the equations for the two other amplitudes are obtained by cyclic permutations and $\mu$ measures the distance from onset $\mu \equiv\left(R-R_{c}\right) / R_{c}$ [Swift, 1984; Soward, 1985]. (The overbar stands for the complex conjugate.) The term $v \bar{A}_{2} \bar{A}_{3}$, assumed to be small, accounts for the resonance of the wavevectors of the three hexagon modes. The coefficients $h_{2}$ and $h_{3}$ determine the interaction between a set of rolls and the set rotated by $+60^{\circ}$ and $-60^{\circ}$ respectively. When rotation is absent, clockwise and anticlockwise rotations are equivalent, so that $h_{2}=h_{3}$ and Eq. (2) reduces to the usual normal form for hexagon patterns. On the contrary, rotation $(T \neq 0)$ breaks the chiral symmetry and implies $h_{2}=h+\nu$ and $h_{3}=h-\nu$, where $h$ indicates the transversal coupling coefficient (without rotation) and $\nu \propto T$. Therefore, for $T \neq 0$ and $v=0$ the BH model is recovered [Busse \& Heikes, 1980]. Invariance arguments require that $v, g$ and $h$ must be even and $\nu$ odd functions of $T$. The bifurcation diagram of Eq. (6) is showed in Fig. 2.

For the model considered the coefficients in the last expression take the form:

$$
\begin{aligned}
v & =\frac{\gamma}{k^{2} R_{c}} \frac{3 \pi^{3}}{\left[\left(k^{2}+4 \pi^{2}\right)^{3}-k^{2} R_{c}+4 \pi^{2} T^{2}\right]} \\
g & =\frac{1}{2 k^{2} R_{c}}\left[\frac{\pi^{4}}{16 \pi^{4}+T^{2}}+\frac{\pi^{6}}{32\left(k^{2}+\pi^{2}\right)^{3}-2 k^{2} R_{c}+2 \pi^{2} T^{2}}\right] \\
h & =\frac{1}{k^{2} R_{c}}\left[\frac{\pi^{4}}{2\left(16 \pi^{4}+T^{2}\right)}+\frac{2 \pi^{6}}{\left(3 k^{2}+4 \pi^{2}\right)^{3}-3 k^{2} R_{c}+4 \pi^{2} T^{2}}+\frac{2 \pi^{6}}{\left(k^{2}+4 \pi^{2}\right)^{3}-k^{2} R_{c}+4 \pi^{2} T^{2}}\right] \\
\nu & =\frac{3 \sqrt{3} \pi^{4} T}{R_{c}}\left[\frac{1}{\left(3 k^{2}+4 \pi^{2}\right)^{3}-3 k^{2} R_{c}+4 \pi^{2} T^{2}}-\frac{1}{\left(k^{2}+4 \pi^{2}\right)^{3}-k^{2} R_{c}+4 \pi^{2} T^{2}}\right]
\end{aligned}
$$

where the time has been rescaled as $t \rightarrow$ $t /\left(k^{2} R_{c}\right)$ (detailed calculations can be found in the Appendix).

Normalized coefficients $v / g_{0}, g / g_{0}, h / g_{0}$ and $\nu / g_{0}$ are depicted as functions of $T$ in Fig. 3 (the reference value $g_{0}=g(T=0)$ is taken for comparison). These dependencies come either explicitly or implicitly through $k_{c}(T)$ and $R_{c}(T)$. The coefficient $v / \gamma g_{0}$ keeps very small values for any $T$ (see Fig. 3). We see in the same figure that $h, g$ and $v$ decrease, while $\nu$ rises almost linearly when $T$ 


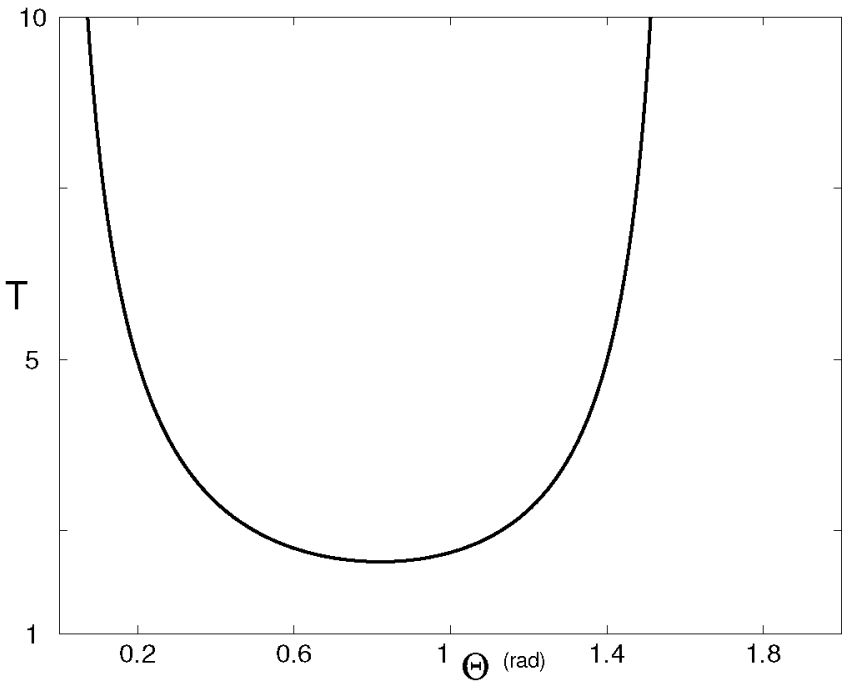

Fig. 1. Instability curve for convective rolls under rotation. The minimum corresponds to the KL instability.

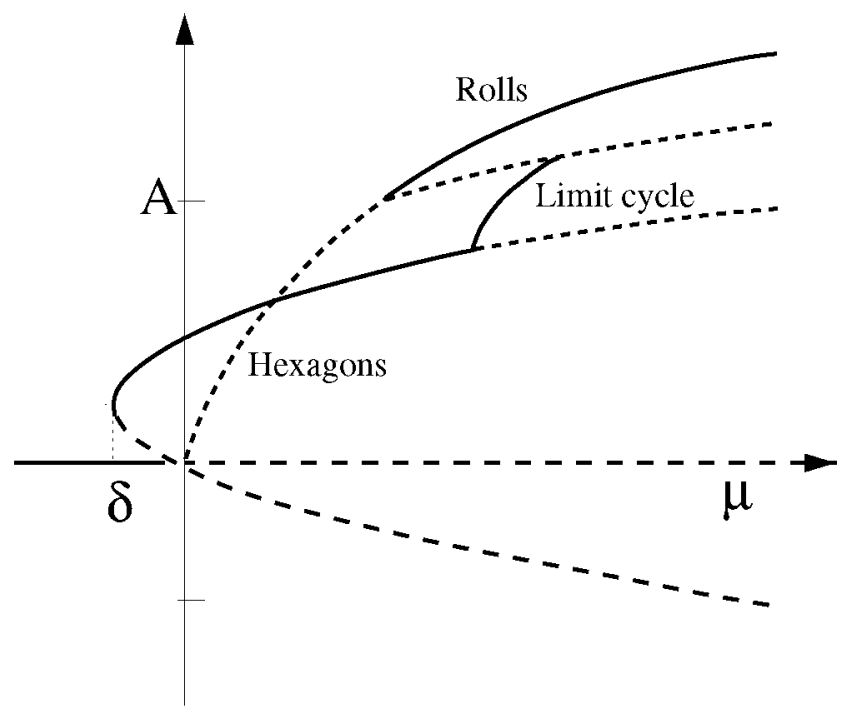

Fig. 2. Bifurcation diagram.

increases, and the condition $h>g$ (stable hexagons) is kept for any rotation.

Stability of Eq. (6) has been studied by several authors [Swift, 1984; Soward, 1985; MillánRodríguez et al., 1992; Sain \& Riecke, 2000]. The stationary solutions are hexagons $H_{1}=H_{2}=H_{3}=$ $H$ with $H=(v+\sqrt{v+4(g+2 h) \mu}) / 2(g+2 h)$, rolls $H_{1}=R, H_{2}=H_{3}=0$ with $R=\sqrt{\mu / g}$ and a limit cycle. (A mixed-mode solution, $A_{1} \neq A_{2}=A_{3} \neq 0$, also exists, but it is always unstable.) The resonant quadratic term $v \bar{A}_{2} \bar{A}_{3}$ breaks the symmetry $A \leftrightarrow-A$, thus breaking the heteroclinic connection
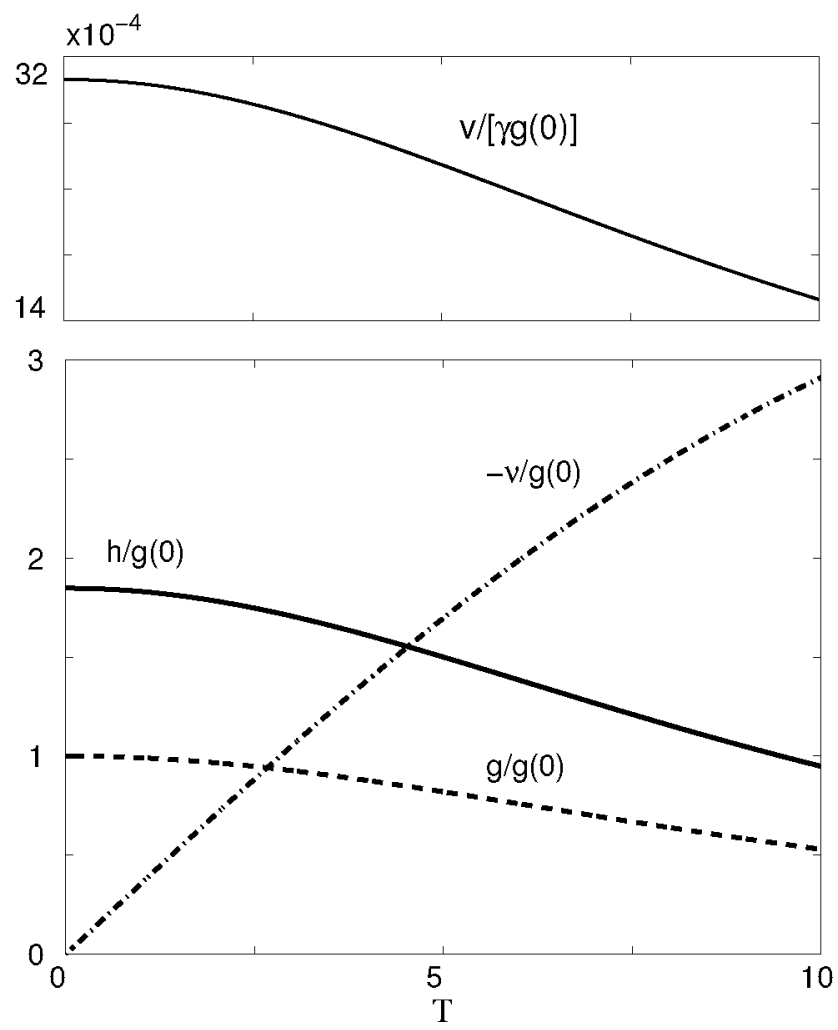

Fig. 3. Coefficients in the normal form as functions of $T$.

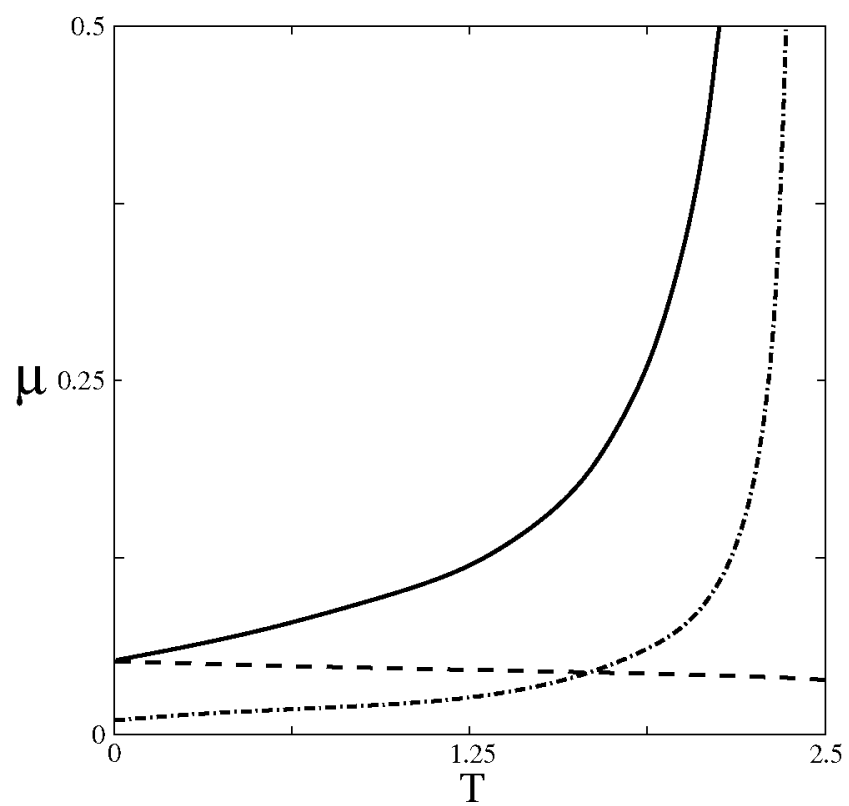

Fig. 4. Stability regions in a $(\mu, T)$ plane. Rolls are stable above the curve labeled Roll. Below the line labeled Hex hexagons are stable. Above this line and to the right of line Het oscillating hexagons are stable.

characteristic of the BH model into a very slow limit cycle [Swift, 1984; Soward, 1985].

A standard linear stability analysis around stationary solutions gives stable hexagons within 


$$
-\frac{v^{2}}{4(g+2 h)} \leq \mu \leq \mu_{H}=\frac{v^{2}(2 g+h)}{(h-g)^{2}}
$$

and stable rolls for

$$
\mu \geq \mu_{R}=\frac{v^{2} g}{(g-h-\nu)(g-h+\nu)}
$$

The hexagons become unstable towards a Hopf bifurcation with $\omega_{c}=2 \sqrt{3} \nu\left(v^{2} /(h-g)^{2}\right)$ at the value $\mu=\mu_{H}$. The branch of oscillating hexagons remains until $\mu=\mu_{\text {het }}$, in which an unstable heteroclinic connection involving the three modes appears.

Existence conditions result in the stability diagram depicted in Fig. 4. Hexagons are stable below the line labeled Hex, rolls above the line Rolls and oscillating hexagons can appear in the region below Het and above Hex. Though $\mu_{H}$ does not depend explicitly on $\nu$ it still has a dependence on $T$ through coefficients. This leads to a reduction of this threshold as rotation $T$ increases, in agreement with the curve computed numerically from hydrodynamic equations reported in [Riecke et al., 2000 . It is worth noticing that rolls are never stable for $|\nu| \geq \nu_{\mathrm{KL}} \equiv h-g$. When the value $\nu_{\mathrm{KL}}$ is exceeded a KL-like instability appears. Nevertheless the limit cycle survives even very far from threshold, but the stability curves Het and Roll tend asymptotically to the associated value $T_{\mathrm{KL}}$ when $\mu$ is increased. After some critical value is reached hexagons bifurcate to oscillating hexagons. This value is determined by $\mu_{R}=\mu_{H}$, thus leading to $\left|\nu_{H}\right|=\left((h+g)(h-g)^{2}\right) / h+2 g$ [Echebarria \& Riecke, 2000]. Investigations of Eq. (6) shows that defects in oscillating hexagons have a complex dynamics (defect chaos) in the region between the curves Hex and Het [Echebarria \& Riecke, 2000]. Thus, complex motion of defects would be reachable even for small heating and rotation.

\section{Wavenumber Perturbations}

Apart from the usual diffusive term, spatial dependence enters also through quadratic terms in Eq. (6) [Gunaratne et al., 1994; Echebarria \& Pérez-García, 1998]. Using symmetry arguments one arrives at the equations

$$
\begin{aligned}
\partial_{t} A_{1}= & \mu A_{1}+v \bar{A}_{2} \bar{A}_{3}-g\left|A_{1}\right|^{2} A_{1} \\
& -(h+\nu)\left|A_{2}\right|^{2} A_{1}-(h-\nu)\left|A_{3}\right|^{2} A_{1} \\
& +\xi_{0}^{2} \partial_{x_{1}}^{2} A_{1}+i \alpha_{1}\left[\bar{A}_{2} \partial_{x_{3}} \bar{A}_{3}+\bar{A}_{3} \partial_{x_{2}} \bar{A}_{2}\right] \\
& +i \alpha_{2}\left[\bar{A}_{2} \partial_{x_{3}} \bar{A}_{3}-\bar{A}_{3} \partial_{x_{2}} \bar{A}_{2}\right] \\
& +i \beta\left[\bar{A}_{2} \partial_{\tau_{3}} \bar{A}_{3}-\bar{A}_{3} \partial_{\tau_{2}} \bar{A}_{2}\right]
\end{aligned}
$$

as discussed in [Echebarria \& Riecke, 2000]. Here $\partial_{x_{i}}$ and $\partial_{\tau_{i}}$ denote the gradient along and perpendicular to the direction of rolls with amplitude $A_{i}$ $\left(\partial_{x_{i}}=\hat{n}_{i} \cdot \nabla, \partial_{\tau_{i}}=\hat{\tau}_{i} \cdot \nabla, \hat{n}_{i} \perp \hat{\tau}_{i}\right)$, respectively. (Notice that the resonant interaction makes needless the usual anisotropic Newell-Whitehead scaling [Pismen \& Nepomnnyashchy, 1993 ].) The chiral symmetry breaking modifies the quadratic nonlinear terms through the coefficient $\alpha_{2}$, which is an odd function of $T$. The remaining spatial coefficients $\left(\xi_{0}^{2}\right.$, $\alpha_{1}$ and $\beta$ ) have to be even functions of $T$.

We have derived spatial coefficients for the model equation (2) by a multiple-scale analysis as indicated in the Appendix. Their explicit expressions are

$$
\begin{aligned}
\xi_{0}^{2} & =\frac{12\left(k^{2}+\pi^{2}\right)^{2}}{R_{c}} \\
\alpha_{1} & =-\frac{\gamma}{k^{2} R_{c}} \frac{3 \pi^{3}\left[\left(k^{2}+4 \pi^{2}\right)^{2}\left(5 k^{2}+4 \pi^{2}\right) q-2 k^{2} R_{c}+4 \pi^{2} T^{2}\right]}{k\left[\left(k^{2}+4 \pi^{2}\right)^{3}-k^{2} R_{c}+4 \pi^{2} T^{2}\right]^{2}} \\
\alpha_{2} & =\frac{\gamma}{k^{2} R_{c}} \frac{3 \sqrt{3} k^{3} \pi R_{c} T}{2\left[\left(k^{2}+4 \pi^{2}\right)^{3}-k^{2} R_{c}+4 \pi^{2} T^{2}\right]^{2}} \\
\beta & =-\frac{\gamma}{k^{2} R_{c}} \frac{\sqrt{3} \pi^{3}\left[3\left(k^{2}+4 \pi^{2}\right)^{2}\left(5 k^{2}+4 \pi^{2}\right)-7 k^{2} R_{c}+12 \pi^{2} T^{2}\right]}{k\left[\left(k^{2}+4 \pi^{2}\right)^{3}-k^{2} R_{c}+4 \pi^{2} T^{2}\right]^{2}}
\end{aligned}
$$

The linear correlation length $\xi_{0}^{2}$ diminishes when $T$ increases in qualitative agreement (though important differences still exist) with experimental findings in [Bajaj et al., 1998] (see Fig. 5). The remaining parameters are quadratic, proportional to NB effects and therefore assumed to be small. For the sake of comparison we have drawn $\alpha_{1} / v$ (dashed line), $\alpha_{2} / v$ (full line) and $\beta / v$ (dot-dashed line) as functions of $T$ in Fig. 5 . 

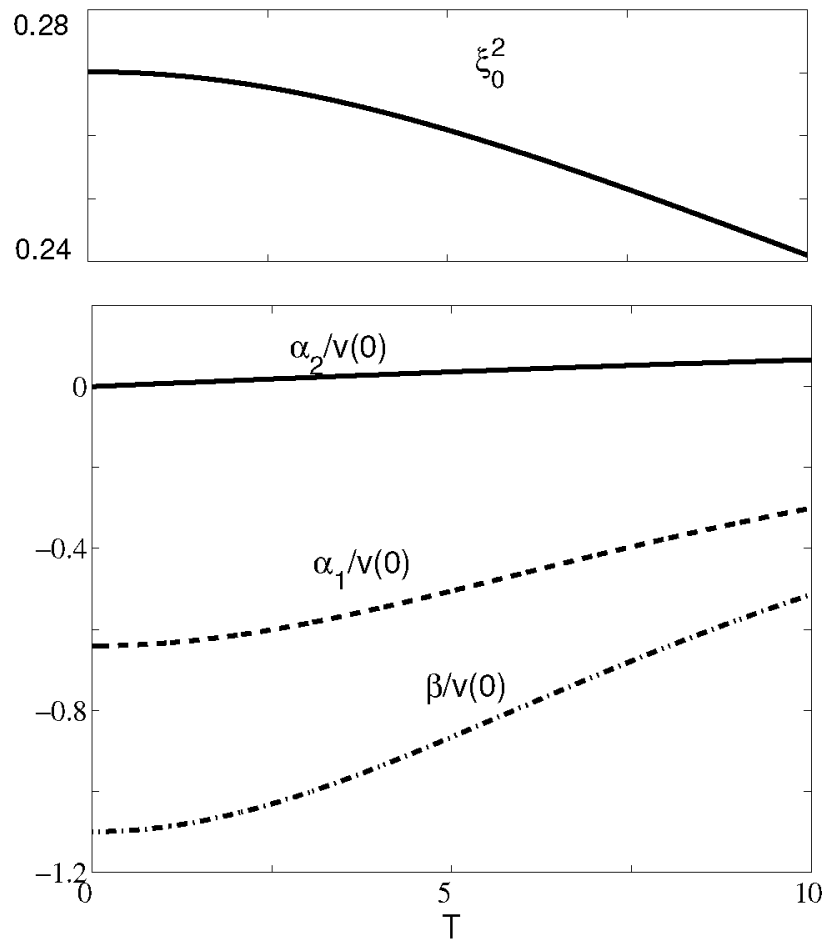

Fig. 5. The coefficients (a) $\xi_{0}^{2}$, (b) $\alpha_{1} / v, \alpha_{2} / v$ and $\beta / v$ as functions of $T$.

As for coefficients in the normal form the dependence of $\alpha_{1}$ and $\beta$ on $T$ is relatively intricate, while $\alpha_{2}$ varies almost linearly with $T$. Moreover $\alpha_{2}$ is very small. The other two coefficients $\alpha_{1}$ and $\beta$ are of the same order than $v$. When rotation is absent $(T=0)$ we get $\alpha_{1} / v=-0.64$, so that the corresponding term contributes substantially to the stability of the pattern.

With these spatial terms hexagons are given by

$$
H=\frac{\left(v+2 q \alpha_{1}\right)+\sqrt{\left(v+2 q \alpha_{1}\right)^{2}+4(g+2 h)\left(\mu-\xi_{0}^{2} q^{2}\right)}}{2(g+2 h)}
$$

which are stable provided the following relationships are fulfilled

$$
\begin{aligned}
u & =2 H^{2}(g-h)+2\left(v+2 q \alpha_{1}\right) H>0 \\
m & =2 H^{2}(g+2 h)-\left(v+2 q \alpha_{1}\right) H>0
\end{aligned}
$$

These conditions determine two curves that do not depend explicitly either on $\nu$ or on $\alpha_{2}$, although rotation $T$ enters into these expression through coefficients. Similarly, rolls are of the form

$$
R=\sqrt{\frac{\mu-\xi_{0}^{2} q^{2}}{g}}
$$

Their stability is bounded by the condition

$$
R^{2}(g-h-2 \nu)+\left(v+2 q \alpha_{1}\right) R>0
$$

We shall comment on the ensuing stability diagrams at the end of this section.

\subsection{Sideband instabilities}

Distorsions involving spatial modulations over distances much larger than the basic wavelength are governed by marginal phase modes, ruled through a phase equation. Let us recall briefly the procedure to get it for stripes. An amplitude of slightly distorted stripes can be split into $A=(R+r)$ $\exp i(q x+\phi)$. After replacing this expression into Eq. (10) one arrives at two coupled equations for the amplitude $r$ and the phase $\phi$. The former is enslaved under the slow phase mode resulting in a diffusion equation known as phase equation, which should be kept invariant under reversing $T \rightarrow-T, z \rightarrow-z$. Therefore, the diffusion coefficient must contain a term independent of the rotation sign plus a term proportional to $T$. This fact modifies the sideband instability regions for stripes under rotation. (The interested reader can find detailed calculations by Friedrich [1993].)

For hexagons, slight perturbations can be written as $A_{i}=\left(H+r_{i}\right) \exp \left(i \mathbf{q} \cdot \mathbf{x}_{\mathbf{i}}+\phi_{i}\right)$. Far from the Hopf bifurcation the dynamics is governed by two independent phase components $\phi=\left(\phi_{x}, \phi_{y}\right)$ related to the translationally invariant modes in a hexagonal lattice by $\phi_{x}=-\left(\phi_{2}+\phi_{3}\right)$ and $\phi_{y}=$ $-\left(\phi_{2}-\phi_{3}\right) / \sqrt{3}$. Without rotation, they evolve according to the expression [Lauzeral et al., 1993; Hoyle, 1995; Echebarria \& Pérez-García, 1998]

$$
\partial_{t} \phi=A \nabla^{2} \phi+B \nabla(\nabla \cdot \phi)
$$

build up with the two isotropic second-order spatial operators acting on a planar field $\phi$. In absence of rotation $\left(\nu=\alpha_{2}=0\right)$ Eq. (17) can be diagonalized by taking $\phi=\phi_{l}+\phi_{t}$, such that $\nabla \times \phi_{l}=\mathbf{0}$ and $\nabla \cdot \phi_{t}=0$. This leads to the uncoupled diffusion equations $\partial_{t} \phi_{l}=(B+A) \nabla^{2} \phi_{l}$ and $\partial_{t} \phi_{t}=A \nabla^{2} \phi_{t}$. Hexagons become unstable whenever $(B+A)$ or $A$ become negative. After introducing the explicit expressions for $A$ and $B$ given in [Echebarria \& Pérez-García, 1998] with coefficients (7) and (11) these conditions lead to the egg-shaped almost-closed curves drawn in Fig. 6. ${ }^{1}$ (The full

\footnotetext{
$\overline{{ }^{1} \text { In Figs. } 6 \text { and } 7 \text { the exaggerated value } \gamma=1000}$ has been taken to have a sufficiently broad stability region.
} 


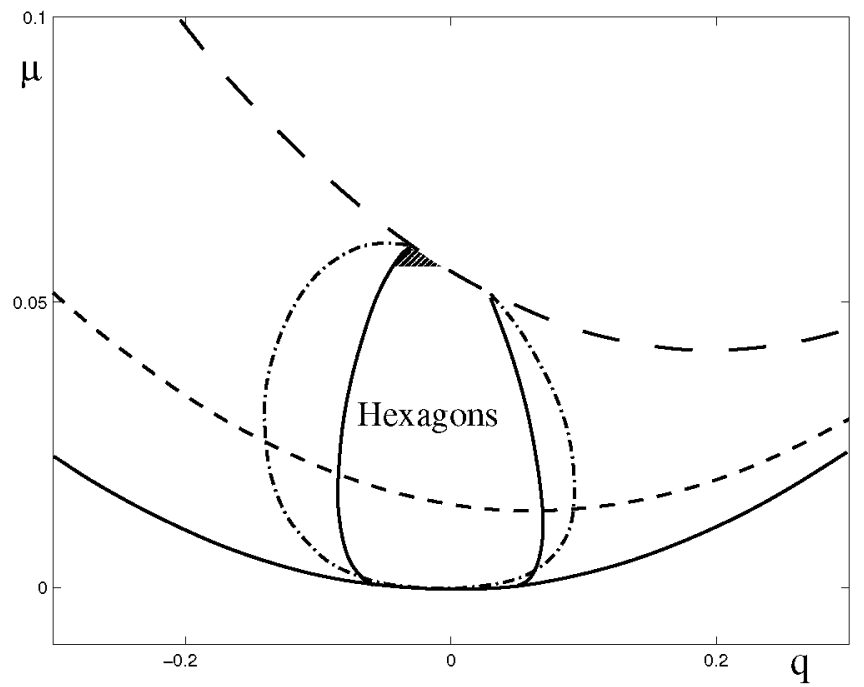

Fig. 6. Amplitude and phase instability curves for $T=0$. Hexagons are stable inside the shaded region.

line corresponds to $A=0$ and the dot-dashed line to $(A+B)=0$.) Hexagons are stable inside the shaded region. The upper amplitude stability curve corresponds to a transcritical bifurcation to rolls which can be reached in a range of wavenumbers. We see that, though small, quadratic spatial terms modify substantially the stability diagrams as shown recently for other systems [Peña \& Pérez-García, 2000]. At variance with these cases the stability region is bent to the left in our model owing to negative values of $\alpha_{1}$ and $\beta$.

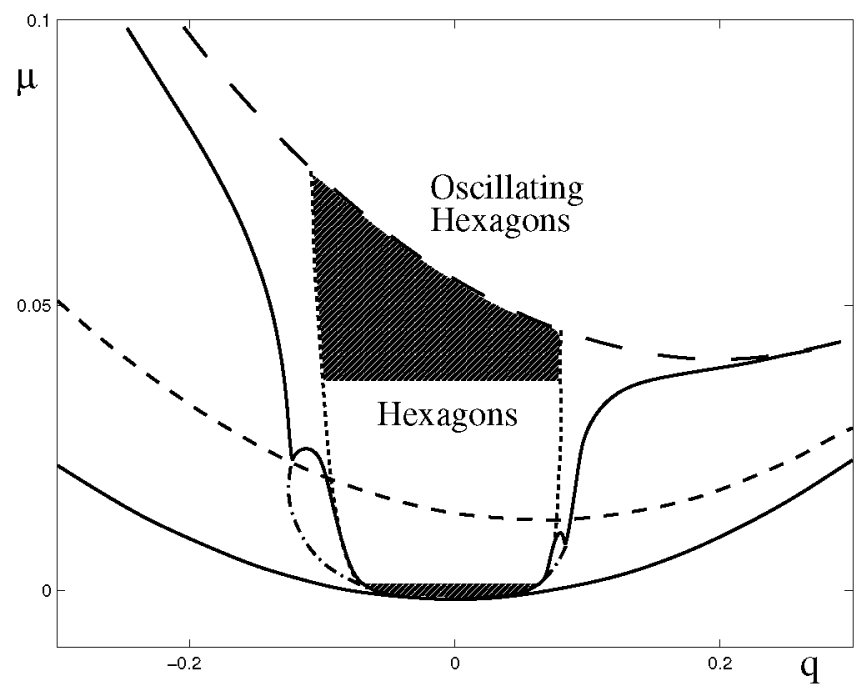

(a)
In the case with rotation $T \neq 0$ the phase equation for hexagons has to include new terms. Similarly to the stripe case, this equation must remain invariant under reflection $T \rightarrow-T, z \rightarrow-z$. Thus the phase equation for hexagons arising from rotating convection takes the form

$$
\begin{aligned}
\partial_{t} \phi= & {\left[A+C(T) \mathbf{e}_{z} \times\right] \nabla^{2} \boldsymbol{\phi} } \\
& +\left[B+D(T) \mathbf{e}_{z} \times\right] \nabla(\nabla \cdot \phi)
\end{aligned}
$$

in which $C$ and $D$ are odd functions of $T$. From generalized Ginzburg-Landau equations (10) the four coefficients in the last expression are found to be [Echebarria \& Riecke, 2000]:

$$
\begin{aligned}
A= & \frac{\xi_{0}^{2}}{4}+\frac{1}{u^{2}+w^{2}}\left\{\frac{H^{2} u}{4}\left[3 \alpha_{2}^{2}+\left(\alpha_{1}-\sqrt{3} \beta\right)^{2}\right]\right. \\
& \left.-q^{2} \xi_{0}^{4} u-H w q \xi_{0}^{2} \sqrt{3} \alpha_{2}\right\} \\
B= & \frac{\xi_{0}^{2}}{2}-\frac{2 q^{2} \xi_{0}^{4}}{m}-\frac{H^{2}}{m} \alpha_{1}\left(\alpha_{1}+\sqrt{3} \beta\right) \\
& +\frac{H \xi_{0}^{2}}{m} q\left(3 \alpha_{1}+\sqrt{3} \beta\right) \\
C= & \frac{-1}{u^{2}+w^{2}}\left\{\sqrt{3} u H q \alpha_{2} \xi_{0}^{2}+\frac{1}{4} H^{2} w\left(3 \alpha_{2}^{2}\right.\right. \\
& \left.\left.+\left(\alpha_{1}-\sqrt{3} \beta\right)^{2}\right)-w q^{2} \xi_{0}^{4}\right\}
\end{aligned}
$$

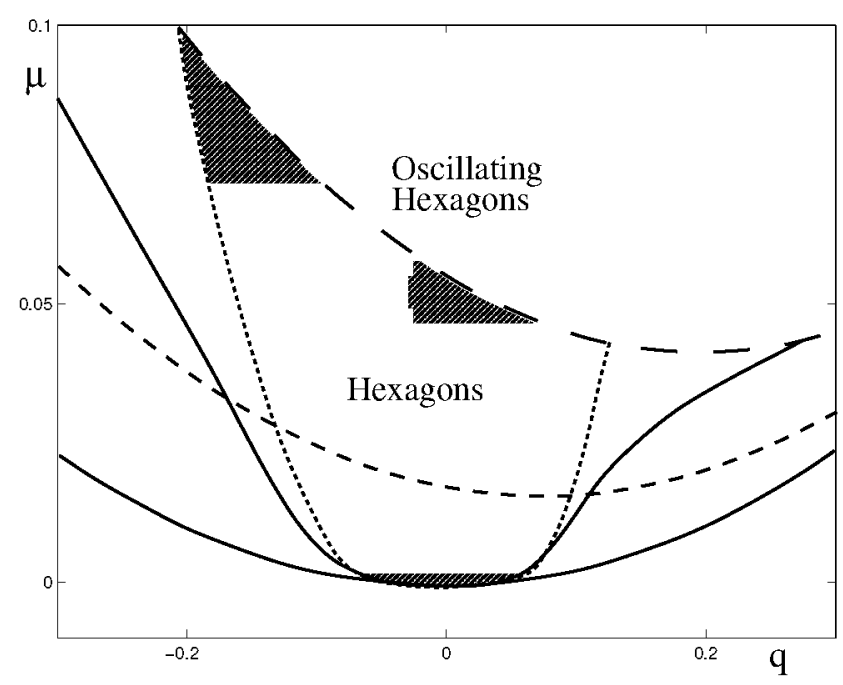

(b)

Fig. 7. Amplitude and phase instability curves for (a) $T=0.1$ and (b) $T=0.9$. The phase instability curve (continuous line) coincides in a range of values with the dotted curve issued from a general stability analysis in a range of $\mu$. Outside the shaded region hexagons become oscillatory. 


$$
D=\frac{\sqrt{3} \alpha_{2}\left(H^{2} \alpha_{1}-q H \xi_{0}^{2}\right)}{m}
$$

with $H, u$ and $m$ given in Eqs. (12)-(14)and $w=$ $2 \sqrt{3} H^{2} \nu$. The eigenvalue problem related to the phase equation (18) have been discussed under some special limits, but the general stability curves have to be obtained numerically.

In Fig. 7 we represent the amplitude and phase instability curves for two different rotations ( $T=$ 0.1 and $T=0.9$ ). The solid bottom and the longdashed line correspond to the saddle-node bifurcation to hexagons and oscillating hexagons, respectively. The dot-dashed line and the continuous is the stability boundary of phase perturbations. Even for small chiral symmetry breaking $(T=0.1)$ the stability diagram changes drastically as seen in Fig. 7(a). The left stability curve does not merge with the amplitude curve $u=0$ (Eq. 13), whereas the right stability curve intersects the line $u=0$ close to its minimum.

To complete this analysis, arbitrary perturbations acting on hexagons $A_{i}=\left(H+h_{i}\right) \exp (\mathbf{q} \cdot \mathbf{x}+$ $\left.\phi_{i}\right), i=1,2,3$ are introduced in Eq. (10). After expanding $h_{i}$ in normal modes $r_{i} \exp i(\mathbf{Q} \cdot \mathbf{x}+\sigma t)$ and linearizing, the resulting eigenvalue problem is solved numerically.

When rotation is absent the most dangerous eigenvalues correspond to phase modes, so that stability limits coincide with phase stability curves (Fig. 6). Nevertheless, in the general case $(T \neq 0)$ translational modes can destabilize hexagons not only by longwave instabilities but also through short-wave modes. For some parameter values the real part of two complex conjugate eigenvalues become positive, so that a Hopf bifurcation to oscillating hexagons takes place [Echebarria \& Riecke, 2000]. The general stability analysis gives the dashed lines in Fig. 7. For $T$ and $\mu$ small $[T=0.1$, Fig. $7(\mathrm{a})]$ one remarks that these lines coincide with those given by a phase stability analysis (continuous lines), while they diverge when $\mu$ is increased beyond a threshold value [Echebarria \& Riecke, 2000].

\section{Conclusions}

The model proposed in this paper constitutes a schematic tool for studying rotating convection. It is based on the linear kernel of Rayleigh-Bénard convection plus some terms accounting for noBoussinesq (NB) contributions, rotation and idealized boundary conditions. For vanishing NB effects, rotating convection displays a Küppers-Lortz (KL) instability. With NB effects included the preferred pattern near onset is hexagonal, but the underlying amplitudes can become oscillatory after some rotation threshold has been exceeded. We calculate the evolution equations for these amplitudes as well as the dependence of their coefficients on rotation. The resulting stability curves agree qualitatively with that found by other authors using a generalized Swift-Hohenberg model [Millán-Rodríguez et al., 1992; Sain \& Riecke, 2000] or by a numerical approach [Echebarria \& Riecke, 2000].

Rotation adds up new terms in the phase dynamics of hexagons. Consequently, the stability diagrams against long-wavelength perturbations depend on rotation. But this analysis has to be completed with a general stability analysis when rotation is present. Let us mention that the present analysis could be extended to deal with the interesting defect chaos of oscillating hexagons [Echebarria \& Riecke, 2000].

Thus this model equation contains some interesting features found in rotating convection, so that it could be evolved numerically as a prospective step before launching the computationally demanding task of simulations of the full fluid and heat equations. These integrations could also be used to validate the stability curves found through Eq. (10).

Although qualitative, our results point to rotating convection under NB conditions as a good candidate to study spatiotemporal complex motions, because this system can be dealt with a combination of analytical and numerical techniques, and experiments seem feasible [Bodenschatz et al., 2000]. In particular, it should be interesting to determine defect chaos for hexagons, very likely different from the domain wall chaos found for stripes [Tu \& Cross, 1992].

\section{Acknowledgments}

The authors gratefully acknowledge helpful discussions with B. Echebarria (Barcelona), H. Mancini, B. Peña (Pamplona) and G. Lebon (Liège). This work was partially supported by the DGICYT (Spanish Government) Grant No. PB98-0578, the PIUNA (Universidad de Navarra). S. Madruga acknowledges the financial support from "Asociación de Amigos de la Universidad de Navarra" and ICOPAC Programme HR-PN-CT-2000-0136, funded by the European Union. 


\section{References}

Assenheimer, M. \& Steinberg, V. [1996] "Observation of coexisting upflow and down-flow hexagons in Boussinesq Rayleigh-Bénard convection," Phys. Rev. Lett. 76, 756-759.

Bajaj, K. M. S., Cannell, D. S. \& Ahlers, G. [1997] "Competition between spiral-defect chaos and rolls in Rayleigh-Bénard convection," Phys. Rev. E55, R4869-R4872.

Bajaj, K. M. S., Liu, J., Naberhuis, B. \& Ahlers, G. [1998] "Square patterns in Rayleigh-Bénard convection with rotation about a vertical axis," Phys. Rev. Lett. 81, 806-809.

Bodenschatz, E., Pesch, W. \& Ahlers, G. [2000] "Recent developments in Rayleigh-Bénard convection," Annual Review of Fluid Mechanics, Vol. 32 (Annual Reviews, Palo Alto, USA), pp. 709-778.

Busse, F. [1967] "The stability of finite amplitude cellular convection and its relations to an extremum principle," J. Fluid Mech. 30, 625-649.

Busse, F. H. \& Heikes, K. E. [1980] "Convection in a rotating layer: A simple case of turbulence," Science $208,173-175$.

Chandrasekhar, S. [1961] Hydrodynamic and Hydromagnetic Stability (Oxford University Press, Oxford).

Ciliberto, S., Pampaloni, E. \& Pérez-García, C. [1988] "Competition between different symmetries in convective patterns," Phys. Rev. Lett. 61, 1198-19201.

Cross, M. C., Meiron, D. \& Tu, Y. [1994] "Chaotic domains: A numerical investigation," Chaos 4, 607-619.

Echebarria, B. \& Pérez-García, C. [1998] "Phase instabilities in hexagonal patterns," Europhys. Lett. 43, $35-41$.

Echebarria, B. \& Riecke, H. [2000] "Stability of oscillating hexagons in rotating convection," Physica D143, $97-108$.

Fantz, M., Friedrich, R., Bestehorn, M. \& Haken, H. [1993] "Pattern formation in rotating Bénard convection," Physica D61, 147-154.

Friedrich, R. [1993] "Phase diffusion equation for roll patterns of systems lacking reflectional symmetry," $Z$. Phys. B92, 129-131.

Gunaratne, G., Ouyang, Q. \& Swinney, H. L. [1994] "Pattern formation in the presence of symmetries," Phys. Rev. E50, 2802-2820.

Hoyle, R. [1995] "Nonlinear phase diffusion equations for the long-wave instabilities in hexagons," Appl. Math. Lett. 9, 81-85.

Küppers, G. \& Lortz, D. [1969] "Transition from laminar convection to thermal turbulence in a rotating fluid layer," J. Fluid Mech. 35, 609-620.

Lauzeral, J., Metens, S. \& Walgraef, D. [1993] "On the phase dynamics of hexagonal patterns," Europhys. Lett. 24, 707-712.
Millán-Rodríguez, J., Pérez-García, C, Bestehorn, M., Neufeld, M. \& Friedrich, R. [1992] "Pattern formation in convection of rotating fluids with broken vertical symmetry," Phys. Rev. A46, 4729-4735.

Morris, S. W., Bodenschatz, E., Cannell, D. S. \& Ahlers, G. [1993] "Spiral defect chaos in large aspect ratio Rayleigh-Bénard convection," Phys. Rev. Lett. 71, 2026-2029.

Peña, B. \& Pérez-García, C. [2000] "Selection and competition of Turing patterns," Europhys. Lett. 51, 300-306.

Pismen, L. M. \& Nepomnyashchy, A. A. [1993] "Structure of dislocations in the hexagonal pattern," Europhys. Lett. 24, 461-467.

Ponty, Y., Passot, T. \& Sulem, P. L. [1997] "Pattern dynamics in rotating convection at finite Prandtl number," Phys. Rev. E56, 4162-4178.

Riecke, H., Echebarria, B., Moroz, V. \& Sain, F. [2000] "Instabilities and defect chaos in models for rotating convection," Proc. 18th Symp. Energy Engineering Sciences, Office of Basic Energy Sciences, Department of Energy, USA, pp. 161-168.

Roxin, A. \& Riecke, H. [2002] "Rotating convection in an anisotropic system," Phys. Rev. E65, 046219.

Sain, F. \& Riecke, H. [2000] "Instabilities and spatiotemporal chaos in hexagon patterns with rotation," Physica D144, 124-141.

Segel, L. A. [1965] "The structure of nonlinear cellular solutions to the Boussinesq equations," J. Fluid Mech. $21,345-358$.

Soward, A. M. [1985] "Bifurcation and stability of finite amplitude convection in a rotating layer," Physica D14, 227-241.

Swift, J. W. [1984] "Convection in a rotating fluid layer," Contemporary Mathematics, Vol. 28 (American Mathematical Society, Providence), pp. 435-448.

Tu, Y. \& Cross, M. C. [1992] "Chaotic domain structure in rotating convection," Phys. Rev. Lett. 69, $2515-2519$.

\section{Appendix}

Here we quote briefly the main calculations to find out Eq. (10). Hexagons can be seen as composed of three plane waves of the form

$$
W=\sum_{i=1}^{3}\left(A_{i} \exp i \mathbf{k} \cdot \mathbf{x}+\text { c.c. }\right)
$$

with $\mathbf{k}_{\mathbf{1}}+\mathbf{k}_{\mathbf{2}}+\mathbf{k}_{\mathbf{3}}=\mathbf{0}$. Slight spatial modulations of a perfect hexagonal pattern are included through envelope functions $A_{i}(x, t)$ varying in space at a scale much larger than the basic wavelength.

Expanding the variables and derivatives in terms of a small parameter $\varepsilon$ one gets

$$
W=\varepsilon W^{(1)}+\varepsilon^{2} W^{(2)}+\varepsilon^{3} W^{(3)}+\ldots,
$$




$$
\begin{gathered}
R=R_{c}+\varepsilon R^{(1)}+\varepsilon^{2} R^{(2)} \\
\partial_{t}=\varepsilon^{2} \partial_{\tau}, \quad \nabla=\nabla_{0}+\varepsilon \nabla_{1}
\end{gathered}
$$

The linear operator can be split into

$$
\begin{aligned}
L & =L^{(0)}+\varepsilon L^{(1)}+\varepsilon^{2} L^{(2)} \\
L & =-\nabla^{6}+R \nabla_{h}^{2}-T^{2} \partial_{z}^{2}-2 \gamma(\cos \pi z) \\
L^{(0)} & =-\nabla_{0}^{6}+R_{0} \nabla_{0}^{2}-T^{2} \partial_{z}^{2}-2 \gamma(\cos \pi z) \\
L^{(1)} & =-6\left(\nabla_{0} \nabla_{1}\right) \nabla_{0}^{4}+2 R_{0} \nabla_{0} \nabla_{1}+R_{1} \nabla_{0}^{2} \\
L^{(2)} & =-12\left(\nabla_{0} \nabla_{1}\right)^{2} \nabla_{0}^{2}-3 \nabla_{0}^{4} \nabla_{1}^{2}
\end{aligned}
$$

Now we solve for each order in $\varepsilon$

$$
\begin{aligned}
& O\left(\varepsilon^{1}\right) L^{(0)} W^{(1)}=0 \\
& O\left(\varepsilon^{2}\right) L^{(0)} W^{(2)}+L^{(1)} W^{(1)} \\
& =-\partial_{z}^{2}\left(W^{(1)} \partial_{z} W^{(1)}\right)+T \mathbf{e}_{z}\left(\nabla W^{(1)} \times \nabla_{0} \partial_{z} W^{(1)}\right) \\
& O\left(\varepsilon^{3}\right) L^{(0)} W^{3}+L^{(1)} W^{(2)}+L^{(2)} W^{(1)} \\
& =-\partial_{\tau}\left(W^{(1)}\right)-\partial_{z}^{2}\left(W^{(1)} \partial_{z} W^{(2)}+W^{(2)} \partial_{z} W^{(1)}\right) \\
& \quad+T \mathbf{e}_{z}\left(\nabla_{0} W^{(2)} \times \nabla_{0} \partial_{z} W^{(1)}\right. \\
& \quad+\nabla_{0} W^{(1)} \times \nabla_{0} \partial_{z} W^{(2)} \\
& \left.\quad+\nabla_{1} W^{(1)} \times \nabla_{1} \partial_{z} W^{(1)}\right)
\end{aligned}
$$

Then we determine the solutions $W^{(1)}, W^{(2)}, W^{(3)}$ for each order:

Order $\varepsilon^{\mathbf{1}}$ : This settles the linear problem $L^{(0)} W^{(1)}=0$. Its solutions have the form:

$$
\begin{aligned}
W^{(1)}= & (\sin \pi z+\gamma K \sin 2 \pi z) \\
& \times \sum_{i=1}^{3}\left(A_{11}^{(i)} \exp i \mathbf{k} \cdot \mathbf{x}+\text { c.c. }\right)
\end{aligned}
$$

with

$$
\begin{aligned}
R_{c} & =\frac{\left(k^{2}+\pi^{2}\right)^{3}+\pi^{2} T^{2}}{k^{2}} \\
K & =\frac{1}{\left(k^{2}+4 \pi^{2}\right)^{3}-k^{2} R_{c}+4 \pi^{2} T^{2}}
\end{aligned}
$$

Order $\varepsilon^{2}$ : The solvability condition (Fredholm alternative) gives

$$
R_{1} k^{2} A_{11}^{(1)}+\frac{3}{2} \pi^{3} \gamma K 2 \bar{A}_{11}^{(2)} \bar{A}_{11}^{(3)}+O\left(\gamma^{2}\right)=0
$$

so that

$$
R_{c}=3\left(k^{2}+\pi^{2}\right)^{2}
$$

which together with Eq. (A.3) determine the critical wavevector. The general solution at this order is $W^{(2)}=W_{p}^{(2)}+W_{h}^{(2)}$, i.e. the homogeneous solution $W_{h}^{(2)}$ plus a particular solution $W_{p}^{(2)}$ given by

$$
\begin{aligned}
& W_{p}^{(2)}=\left(\alpha_{0} \sin 2 \pi z+\alpha_{1} \gamma \sin \pi z+\alpha_{2} \gamma \sin 3 \pi z\right) \\
& \times\left(\sum_{i=1}^{3}\left(A_{21}^{i} \exp i \mathbf{k} \cdot \mathbf{x}+\text { c.c. }\right)\right. \\
& +\left(\alpha_{0}^{\prime} \sin 2 \pi z+\alpha_{1}^{\prime} \gamma \sin \pi z+\alpha_{2}^{\prime} \gamma \sin 3 \pi z\right) \\
& \times\left(\sum_{i=1}^{3}\left(A_{22}^{i} \exp 2 i \mathbf{k} \cdot \mathbf{x}+\text { c.c. }\right)\right. \\
& +\left(\alpha_{0}^{\prime \prime} \sin 2 \pi z+\alpha_{1}^{\prime \prime} \gamma \sin \pi z+\alpha_{2}^{\prime \prime} \gamma \sin 3 \pi z\right) \\
& \times\left(\sum_{i=1}^{3}\left(A_{22}^{i j} \exp i\left(\mathbf{k}_{\mathbf{i}}-\mathbf{k}_{\mathbf{j}}\right) \cdot \mathbf{x}+\text { c.c. }\right)\right. \\
& +\left(\alpha_{0}^{\prime \prime \prime} \sin 2 \pi z+\alpha_{1}^{\prime \prime \prime} \gamma \sin \pi z\right. \\
& \left.+\alpha_{2}^{\prime \prime \prime} \gamma \sin 3 \pi z\right) A_{20} \\
& +\gamma \alpha^{R_{1}} \sin 2 \pi z \sum_{i=1}^{3}\left(A_{i} \exp i \mathbf{k} \cdot \mathbf{x}+\text { c.c. }\right) \\
& +\gamma \alpha^{\nabla} \sin 2 \pi z \sum_{i=1}^{3}\left(\nabla A_{i} \exp i \mathbf{k} \cdot \mathbf{x}+\text { c.c. }\right)
\end{aligned}
$$

in which the coefficients useful for us result in

$$
\alpha_{0}^{\prime} A_{22}^{i}=\frac{\pi^{3} A_{11}^{(i) 2}}{32\left(k^{2}+4 \pi^{2}\right)^{3}-2 k^{2} R_{c}+2 \pi^{2} T^{2}}
$$

$$
\alpha_{0} A_{21}^{i}=\frac{4 \pi^{3} \bar{A}_{11}^{(j)} \bar{A}_{11}^{(k)}}{\left(k^{2}+4 \pi^{2}\right)^{3}-k^{2} R_{c}+4 \pi^{2} T^{2}}
$$

$$
\alpha_{0}^{\prime \prime} A_{22}^{i j}=\frac{4 \pi^{3} A_{11}^{(i)} \bar{A}_{11}^{(j)}}{\left(3 k^{2}+4 \pi^{2}\right)^{3}-3 k^{2} R_{c}+4 \pi^{2} T^{2}}
$$$$
\alpha_{0}^{\prime \prime \prime} A_{20}=\frac{\pi\left(\left|A_{11}^{(1)}\right|+\left|A_{11}^{(2)}\right|+\left|A_{11}^{(3)}\right|\right)}{16 \pi^{2}+T^{2}}
$$

$$
\alpha^{R_{1}}=\frac{R_{1} k^{2}}{\left(\left(k^{2}+4 \pi^{2}\right)^{3}-k^{2} R_{c}+4 \pi^{2} T^{2}\right)^{2}}
$$

$$
\alpha^{\nabla}=-\frac{2 R_{c}}{\left(\left(k^{2}+4 \pi^{2}\right)^{3}-k^{2} R_{c}+4 \pi^{2} T^{2}\right)^{2}}
$$


Order $\varepsilon^{3}$ : The Fredholm alternative ends in

$$
\begin{aligned}
\frac{1}{k^{2} R_{c}} \partial_{\tau} A_{11}^{(1)}= & \frac{R_{2}}{R_{c}} A_{11}^{(1)}+\frac{R_{1}}{R_{c}} A_{11}^{(1)}+\frac{v}{2} \bar{A}_{11}^{(2)} \bar{A}_{11}^{(3)} \\
& -g\left|A_{11}^{(2)}\right|^{2} A_{11}^{(1)}-(h+\nu)\left|A_{11}^{(2)}\right|^{2} A_{11}^{(1)} \\
& -(h-\nu)\left|A_{11}^{(3)}\right|^{2} A_{11}^{(1)}+\xi_{0}^{2} \partial_{x_{1}}^{2} A_{11}^{(1)} \\
& +i \alpha_{1}\left[\bar{A}_{11}^{(2)} \partial_{x_{3}} \bar{A}_{11}^{(3)}+\bar{A}_{11}^{(3)} \partial_{x_{2}} \bar{A}_{11}^{(2)}\right] \\
& +i \alpha_{2}\left[\bar{A}_{11}^{(2)} \partial_{x_{3}} \bar{A}_{11}^{(3)}-\bar{A}_{11}^{(3)} \partial_{x_{2}} \bar{A}_{11}^{(2)}\right] \\
& +i \beta\left[\bar{A}_{11}^{(2)} \partial_{\tau_{3}} \bar{A}_{1}^{(3)}-\bar{A}_{11}^{(3)} \partial_{\tau_{2}} \bar{A}_{11}^{(2)}\right]
\end{aligned}
$$

where $\partial_{x_{i}}=\hat{n}_{i} \cdot \nabla, \partial_{\tau_{i}}=\hat{\tau}_{i} \cdot \nabla$ with the unit vectors defined as

$$
\hat{n}_{1}=(1,0) \quad \hat{\tau}_{1}=(0,-1)
$$

$$
\begin{array}{ll}
\hat{n}_{2}=\frac{1}{2}(-1, \sqrt{3}) & \hat{\tau}_{2}=\frac{1}{2}(\sqrt{3}, 1) \\
\hat{n}_{3}=\frac{1}{2}(-1,-\sqrt{3}) & \hat{\tau}_{3}=\frac{1}{2}(-\sqrt{3}, 1)
\end{array}
$$

The amplitude evolution equation is obtained by adding $O\left(A_{i}^{2}\right) \times \varepsilon^{2}+O\left(A_{i}^{3}\right) \times \varepsilon^{3}$ and taking into account that $A_{i}=\varepsilon A_{i}^{(1)}+\varepsilon^{2} A_{i}^{(2)}, A_{i} A_{j}=\varepsilon^{2} A_{11}^{(i)} A_{11}^{(j)}+$ $2 \varepsilon^{3} A_{11}^{(i)} A_{11}^{(j)}+O\left(\varepsilon^{4}\right)$. Finally we arrive at the amplitude equation

$$
\begin{aligned}
\partial_{t} A_{1}= & \mu A_{1}+v \bar{A}_{2} \bar{A}_{3}-g\left|A_{1}\right|^{2} A_{1} \\
& -(h+\nu)\left|A_{2}\right|^{2} A_{1}-(h-\nu)\left|A_{3}\right|^{2} A_{1} \\
& +\xi_{0}^{2} \partial_{x_{1}}^{2} A_{1}+i \alpha_{1}\left[\bar{A}_{2} \partial_{x_{3}} \bar{A}_{3}+\bar{A}_{3} \partial_{x_{2}} \bar{A}_{2}\right] \\
& +i \alpha_{2}\left[\bar{A}_{2} \partial_{x_{3}} \bar{A}_{3}-\bar{A}_{3} \partial_{x_{2}} \bar{A}_{2}\right]+i \beta\left[\bar{A}_{2} \partial_{\tau_{3}} \bar{A}_{3}\right. \\
& \left.-\bar{A}_{3} \partial_{\tau_{2}} \bar{A}_{2}\right]
\end{aligned}
$$

with coefficients

$$
\begin{aligned}
\mu & =\frac{\Delta R}{R_{c}} \\
v & =\frac{\gamma}{k^{2} R_{c}} \frac{3 \pi^{3}}{\left(\left(k^{2}+4 \pi^{2}\right)^{3}-k^{2} R_{c}+4 \pi^{2} T^{2}\right)} \\
g & =\frac{1}{k^{2} R_{c}}\left(\frac{\pi^{4}}{2\left(16 \pi^{4}+T^{2}\right)}+\frac{\pi^{6}}{2\left(32\left(k^{2}+\pi^{2}\right)^{3}-2 k^{2} R_{c}+2 \pi^{2} T^{2}\right)}\right) \\
h & =\frac{1}{k^{2} R_{c}}\left(\frac{\pi^{4}}{2\left(16 \pi^{4}+T^{2}\right)}+\frac{2 \pi^{6}}{\left(\left(3 k^{2}+4 \pi^{2}\right)^{3}-3 k^{2} R_{c}+4 \pi^{2} T^{2}\right)}+\frac{3 \sqrt{3} k^{2} \pi^{4} T}{\left(k^{2}+4 \pi^{2}\right)^{3}-k^{2} R_{c}+4 \pi^{2} T^{2}}\right) \\
\nu & =\tau \frac{1}{k^{2} R_{c}}\left(\frac{3 \sqrt{3} k^{2} \pi^{4} T}{\left(3 k^{2}+4 \pi^{2}\right)^{3}-3 k^{2} R_{c}+4 \pi^{2} T^{2}}-\frac{\left(k^{2}+4 \pi^{2}\right)^{3}-k^{2} R_{c}+4 \pi^{2} T^{2}}{(2}\right) \\
\xi_{0}^{2} & =\frac{12\left(k^{2}+\pi^{2}\right)^{2}}{R_{c}} \\
\alpha_{1} & =-\frac{\gamma}{k^{2} R_{c}} \frac{\left.3 \pi^{3}\left(\left(k^{2}+4 \pi^{2}\right)^{2}\left(5 k^{2}+4 \pi^{2}\right)-2 k^{2} R_{c}+4 \pi^{2} T^{2}\right)\right)}{k\left(\left(k^{2}+4 \pi^{2}\right)^{3}-k^{2} R_{c}+4 \pi^{2} T^{2}\right)^{2}} \\
\beta & =-\frac{\gamma}{k^{2} R_{c}} \frac{\sqrt{3} \pi^{3}\left(3\left(k^{2}+4 \pi^{2}\right)^{2}\left(5 k^{2}+4 \pi^{2}\right)-7 k^{2} R_{c}+12 \pi^{2} T^{2}\right)}{k\left(\left(k^{2}+4 \pi^{2}\right)^{3}-k^{2} R_{c}+4 \pi^{2} T^{2}\right)^{2}} \\
\alpha_{2} & =\frac{\gamma}{k^{2} R_{c}} \frac{2\left(\left(k^{2}+4 \pi^{2}\right)^{3}-k^{2} R_{c}+4 \pi^{2} T^{2}\right)^{2}}{2} \\
\beta &
\end{aligned}
$$

in which the time has been renormalized as $t \rightarrow t /\left(k^{2} R_{c}\right)$. 